\title{
Article
}

\section{Pseudomorphic Growth of a Single Element Quasiperiodic Ultrathin Film on a Quasicrystal Substrate}

Ledieu, J., Hoeft, J., Reid, D., Smerdon, Joe, Diehl, R., Lograsso, T., Ross, A. and McGrath, R.

Available at https://clok.uclan.ac.uk/9500/

Ledieu, J., Hoeft, J., Reid, D., Smerdon, Joe orcid iconORCID: 0000-0002-73878362, Diehl, R., Lograsso, T., Ross, A. and McGrath, R. (2004) Pseudomorphic Growth of a Single Element Quasiperiodic Ultrathin Film on a Quasicrystal Substrate. Physical Review Letters, 92 (13). ISSN 0031-9007

It is advisable to refer to the publisher's version if you intend to cite from the work. http://dx.doi.org/10.1103/PhysRevLett.92.135507

For more information about UCLan's research in this area go to http://www.uclan.ac.uk/researchgroups/ and search for <name of research Group>.

For information about Research generally at UCLan please go to http://www.uclan.ac.uk/research/

All outputs in CLoK are protected by Intellectual Property Rights law, including Copyright law. Copyright, IPR and Moral Rights for the works on this site are retained by the individual authors and/or other copyright owners. Terms and conditions for use of this material are defined in the policies page.

\section{CLoK}

Central Lancashire online Knowledge www.clok.uclan.ac.uk

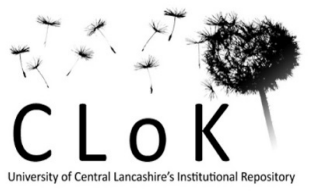




\title{
Pseudomorphic Growth of a Single Element Quasiperiodic Ultrathin Film on a Quasicrystal Substrate
}

\author{
J. Ledieu, J. T. Hoeft, D. E. Reid, and J. A. Smerdon \\ Surface Science Research Centre, The University of Liverpool, Liverpool L69 3BX, United Kingdom \\ R. D. Diehl \\ Department of Physics, Pennsylvania State University, University Park, Pennsylvania 16802, USA
}

T. A. Lograsso and A. R. Ross

Ames Laboratory, Iowa State University, Ames, Iowa 50011, USA

R. McGrath*

Surface Science Research Centre and Department of Physics, The University of Liverpool, Liverpool L69 3BX, United Kingdom

(Received 1 October 2003; published 2 April 2004)

\begin{abstract}
An ultrathin film with a periodic interlayer spacing was grown by the deposition of $\mathrm{Cu}$ atoms on the fivefold surface of the icosahedral $\mathrm{Al}_{70} \mathrm{Pd}_{21} \mathrm{Mn}_{9}$ quasicrystal. For coverages from 5 to 25 monolayers, a distinctive quasiperiodic low-energy electron diffraction pattern is observed. Scanning tunneling microscopy images show that the in-plane structure comprises rows having separations of $S=4.5 \pm$ $0.2 \AA$ and $L=7.3 \pm 0.3 \AA$, whose ratio equals $\tau=1.618 \ldots$ within experimental error. The sequences of such row separations form segments of terms of the Fibonacci sequence, indicative of the formation of a pseudomorphic $\mathrm{Cu}$ film.
\end{abstract}

DOI: 10.1103/PhysRevLett.92.135507

The mathematics of aperiodic order has been an active and fascinating branch of mathematics for many centuries [1,2]. The discovery of quasicrystals [3] in 1982 enabled the study of the physics of aperiodic order [4], and this undertaking has led to significant progress in understanding in many areas including, for example, the behavior of electrons [5], phonons [6] and phason defects [7] in such systems. However, quasicrystals are typically complex ternary alloys, and hence the elucidation of their growth, structure, and physical properties is a difficult undertaking. Therefore, there is significant interest in searching for quasiperiodic systems of reduced complexity and dimensionality.

One route to the formation of such systems is to use surfaces as growth templates. A class of heteroepitaxial systems currently under investigation is that of metal deposition on quasicrystal substrates. There are three possibilities for epitaxial growth in such systems [8]. The first is the formation of a crystalline film with no structural relationship to the substrate. The second is the formation of a crystalline film which is orientationally aligned with the substrate (rotational epitaxy). The third possibility is that of pseudomorphic growth where the film grows in a commensurate structure (necessarily different from its normal elemental crystalline form) in a manner dictated by its interaction with the substrate [9]. This latter growth mode is required for the achievement of a single element quasiperiodic structure.

There have been a number of significant recent studies of such heteroepitaxial systems [10-15]. Two reports of rotational epitaxy have been made. Bolliger et al. [11]
PACS numbers: 61.44.Br, 61.14.Hg, 68.35.Bs, 68.37.Ef

have demonstrated that $\mathrm{Al}$ adsorption on icosahedral Al-Pd-Mn ( $i-\mathrm{Al}-\mathrm{Pd}-\mathrm{Mn})$ results in five face-centered cubic domains of Al, where the [111] axes of the Al nanocrystals are aligned parallel to one of the threefold symmetry axes of the substrate. Fournée et al. [10] have shown that Ag deposition on the fivefold surface of $i$-Al-Pd-Mn results in the growth of hexagonal Ag nanocrystals which grow in five orientations, reflecting the symmetry of the substrate.

There have been three reports of pseudomorphic systems. Cai et al. [12] found the formation of small Al nanoclusters for submonolayer deposition of $\mathrm{Al}$ on $i-\mathrm{Al}-\mathrm{Cu}-\mathrm{Fe}$ at a coverage of $0.04 \mathrm{ML}$. Franke et al. [13] demonstrated the formation of $\mathrm{Sb}$ and $\mathrm{Bi}$ quasiperiodic monolayers on $i$-Al-Pd-Mn and decagonal Al-Ni-Co ( $d$-Al-Ni-Co) by deposition at $570 \mathrm{~K}$ followed by annealing at $820 \mathrm{~K}$. Shimoda et al. [14] reported a $10 \AA$ thick binary Al-Au alloy film having an epitaxial relationship to the substrate produced by deposition of $\mathrm{Au}$ onto the surface in the presence of an In surfactant layer followed by annealing to $700 \mathrm{~K}$.

Finally, in a different class of heteroepitaxial system, Smith et al. [15] reported the formation of a $0.4 \AA$ quasiperiodic superstructure modulation of a $15 \AA$ thin $\operatorname{Ag}(111)$ film on a GaAs(110) substrate formed by deposition at $135 \mathrm{~K}$ followed by annealing to room temperature. This superstructure modulation was used for a real-space study of phasons and dislocations [16].

In this Letter we demonstrate the growth of a pseudomorphic single element multilayer film by deposition of $\mathrm{Cu}$ on the fivefold surface of the $i-\mathrm{Al}-\mathrm{Pd}-\mathrm{Mn}$ at $300 \mathrm{~K}$. The 
quasiperiodic structure within the layers of this ultrathin film is evidenced in low-energy electron diffraction (LEED) patterns and in Fibonacci relationships between rows of $\mathrm{Cu}$ atoms imaged using scanning tunneling microscopy (STM). We show intriguing and unexpected features occurring in the growth process. This work opens up the possibility of the study of the consequences of quasiperiodicity on physical properties independent of chemical composition.

The starting point of these investigations is the preparation of clean surfaces of $\mathrm{Al}_{70} \mathrm{Pd}_{21} \mathrm{Mn}_{9}$ with large flat terraces of micron dimensions and low surface corrugation $(0.3 \AA)$ in an ultra-high-vacuum (UHV) environment. The preparation procedure has been described elsewhere $[17,18]$. LEED was used to establish the degree of surface ordering, and Auger electron spectroscopy (AES) was used to monitor surface cleanliness and composition. Al-Pd-Mn surfaces prepared in this manner have been shown to be essentially ideal terminations of the bulk quasicrystal structure [17-19], and the twodimensional structure of these surfaces has been described as a "Fibonacci pentagrid" [20], with the five principal symmetry axes evident in the arrangement of the dominant structural motifs on the surface.

Deposition of $\mathrm{Cu}$ atoms on the surface was achieved using a simple evaporation source consisting of a tantalum filament around which was wrapped pure $\mathrm{Cu}$ wire. The filament was thoroughly degassed before evaporation and the evaporation flux was found to be very consistent under constant conditions of the control parameters. The adsorption experiments were repeated several times with different $\mathrm{Cu}$ fluxes ranging from $9.0 \times 10^{-3}$ to $4.0 \times$ $10^{-2}$ monolayers (ML) s ${ }^{-1}$ as determined by measuring the fractional area of the surface covered with successive $\mathrm{Cu}$ depositions in the layer-by-layer growth phase described below. The sample was at room temperature during deposition and measurement.

Copper growth on the quasicrystal surface at room temperature was monitored using STM, LEED, and AES. The initial growth up to $8 \mathrm{ML}$ was observed to proceed in a layer-by-layer manner. Figure 1 gives an overview of this process at various stages up to $5.5 \mathrm{ML}$. For submonolayer coverages, shown in Fig. 1(a), small Cu islands form on the surface and as adsorption continues the second layer is observed to begin growing when the first layer is $90 \pm 5 \%$ completed. Figure 1(b) shows the surface at a coverage of $3.8 \pm 0.2 \mathrm{ML}$. Regular shapes at the edges of the growing layer are evident in this figure, along with some linear features within the fourth layer.

Figure 1(c) shows a $700 \times 700 \AA$ image of the film at a coverage of $5.5 \pm 0.2 \mathrm{ML}$. The image reveals a patchwork of islands in the top layer. The edges of the growing layer are straight and meet at well-defined angles, suggestive of well-ordered islands. Figure 1(d) shows AES measurements which were taken during film growth and which show the ratio between the $\mathrm{Cu}_{L V V}$ peak at $60 \mathrm{eV}$ and the $A l_{L V V}$ peak at $68 \mathrm{eV}$ as a function of layer thickness. The

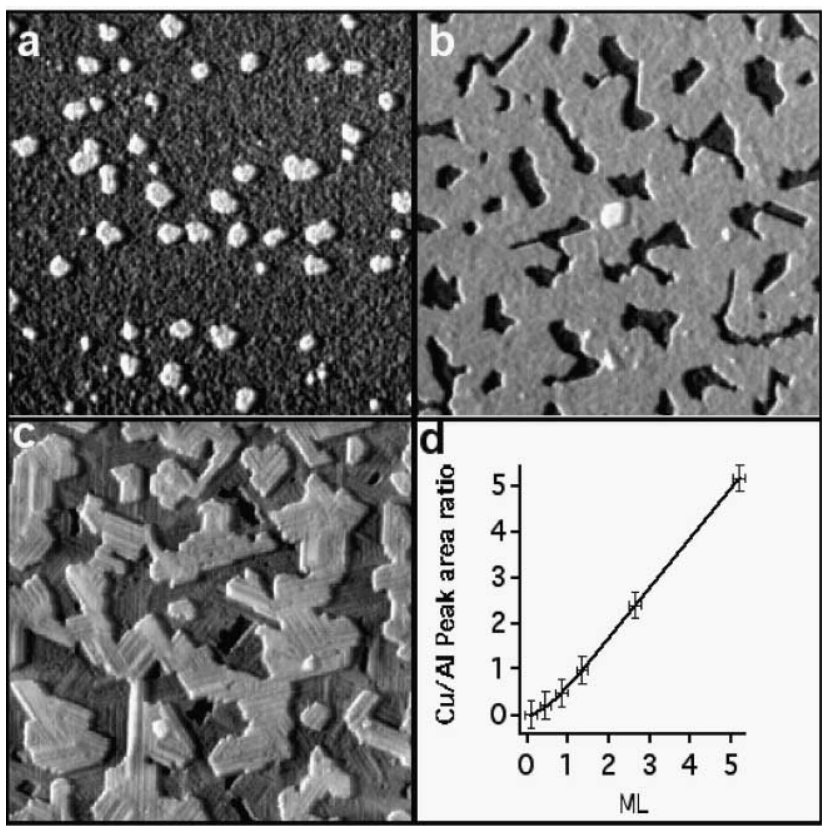

FIG. 1. (a),(b) $500 \times 500 \AA$ STM images of the fivefold surface of Al-Pd-Mn after $\mathrm{Cu}$ deposition at coverages of 0.09 and $3.8 \mathrm{ML}$, respectively. (c) $700 \times 700 \AA$ STM image of the surface at a coverage of 5.5 ML. (d) The ratios of the intensities of the $\mathrm{Cu}_{L V V}(60 \mathrm{eV})$ and $\mathrm{Al}_{L V V}(68 \mathrm{eV})$ Auger peaks as a function of coverage of $\mathrm{Cu}$ (in monolayers).

measurements are consistent with a growing film of pure $\mathrm{Cu}$ with no evidence for alloying with the substrate $[21,22]$. At higher coverage only $\mathrm{Cu}$ peaks are observed in the Auger spectrum.

Figure 2 (a) is a $400 \times 400 \AA$ image at the $5.5 \mathrm{ML}$ coverage. This image shows that the structure consists of rows which are aligned in five orientations. The domain boundaries between the row structures are of two types which are indicated in the figure. The first type occurs when two sets of rows share a vertex point (where the end of each row in one direction meets the end of another at an angle of $108^{\circ}$ ). In the second type the rows in one direction intersect a single row at an angle of $72^{\circ}$. The corrugation across the rows is measured at $0.25 \pm$ $0.05 \AA$ and there are some vacancy defects in the rows. The correlation of the row structure from layer to layer indicates that each layer acts as a template for the subsequent one in the growth process. The observation of the straight island edges suggests a growth mechanism of adsorption and anisotropic diffusion followed by attachment of $\mathrm{Cu}$ atoms at the ends of the rows.

The interrow distances form quasiperiodic sequences having long and short separations. This is illustrated in Fig. 2(b) which is a $100 \times 100 \AA$ region of the surface outlined in Fig. 2(a). Sequences of rows having two distinct interrow spacings are visible. Figure 2(c) shows a height profile between the points indicated by crosses in Fig. 2(b). The average row separations are measured to be $S=4.5 \pm 0.2 \AA$ and $L=7.3 \pm 0.3 \AA$. The ratio of these numbers equals $1.6 \pm 0.1$ which agrees with the golden 


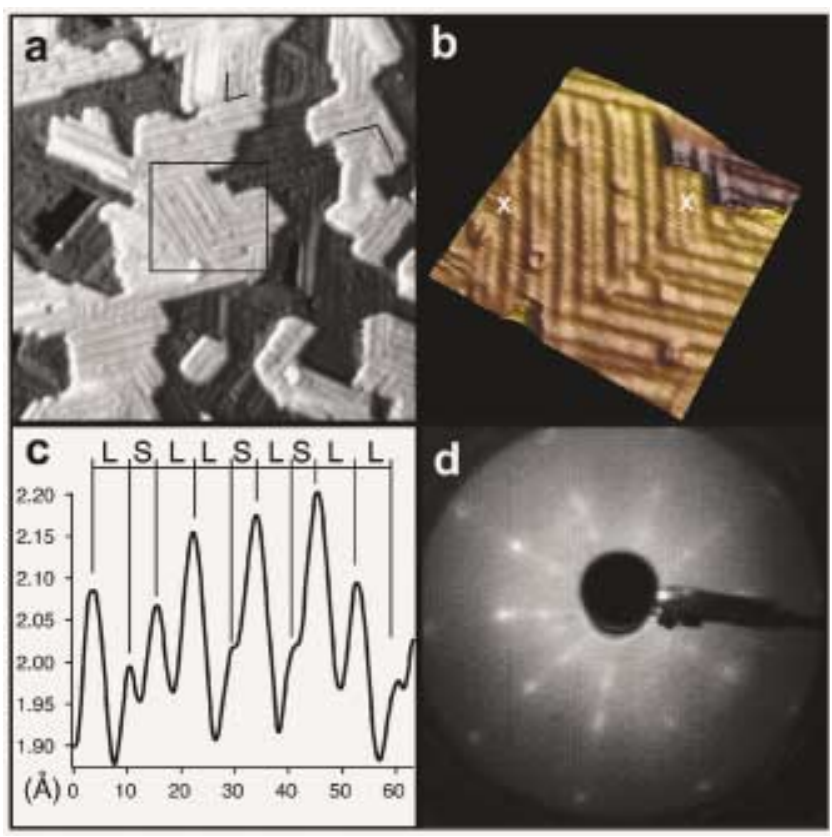

FIG. 2 (color). (a) $400 \times 400 \AA$ STM image of the fivefold surface of Al-Pd-Mn after deposition of $5.5 \mathrm{ML}$ of $\mathrm{Cu}$. (b) $100 \times 100 \AA$ detail from (a). (c) A profile between the points marked with a cross in (b); it demonstrates that the sequence of $\mathrm{Cu}$ rows is quasiperiodic with spacings given by LSLLSLSLL, where $S=4.5 \pm 0.2 \AA$ and $L=7.3 \pm 0.3 \AA$. The ratio of these numbers equals the golden mean $\tau$ within experimental error. (d) LEED pattern (beam energy $50 \mathrm{eV}$ ) corresponding to this phase. The relationships between spot positions are indicative of $\tau$ scaling.

mean $\tau=1.618 \ldots$ within experimental error. The sequence of separations $L S L L S L S L L$ revealed in this height profile is a segment of a term in the Fibonacci sequence. A similar sequence is observed along the adjacent row structure which has a domain boundary at the row intersections. The coherence length of the row structures is determined by these domain walls which arise during the growth process. At the right-hand end of this line a sequence of spacings $S S S$ is observed due to the inclusion of an extra row in the structure [23]. This constitutes the occurrence of a phason defect which is a break in the quasiperiodic ordering of the structure. The atomic structure within the rows is not resolved in these experiments; this could be an indication of a low corrugation in electronic density along the rows.

For layers 1 to 3 the LEED pattern characteristic of the substrate is gradually extinguished, and a new LEED pattern with diffraction spots commensurate with spots on the clean surface pattern emerges. During the growth of the fourth layer there is evidence of an ordered structure emerging in STM images of the film and the LEED pattern becomes well developed. The LEED pattern characteristic of the layered row structure at a coverage of 5.5 ML is shown in Fig. 2(d). The pattern has tenfold symmetric rings of spots whose distances from the central spot [hidden by the electron gun in Fig. 2(d)] exhibit

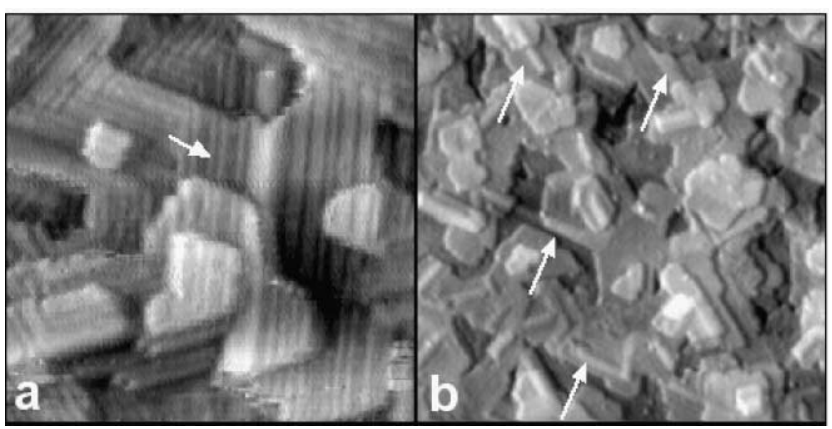

FIG. 3. (a) $350 \times 350 \AA$ STM image of the Al-Pd-Mn surface dosed with $8.7 \mathrm{ML}$ of $\mathrm{Cu}$. The correlation of the row structures across four atomic steps is indicated by the arrow. (b) $800 \times 800 \AA$ image of the surface after adsorption of 11.7 ML. The features highlighted by arrows are discussed in the text.

a $\tau$-scaling relationship indicative of quasicrystalline ordering [24]. There is some smearing of the spots both in the radial and azimuthal directions evident at some beam energies probably due to some disruption of both the domain-domain and row-row correlations. There is no evidence in the LEED pattern for the formation of a crystalline structure, and thus we rule out the possibility that the row structure is a quasiperiodic superstructure modulation of a crystalline structure, as in the case of the $\mathrm{Ag} / \mathrm{GaAs}(110)$ system [15].

At higher coverages the effect of layer growth before previous layer completion becomes more marked. This is illustrated in Fig. 3(a) which shows a $260 \times 260 \AA$ image of the film at an equivalent coverage of $8.7 \pm 0.2 \mathrm{ML}$, where a total of four incomplete layers are visible. The step height from layer to layer is constant at $1.9 \pm 0.1 \AA$, indicating that the film is periodic in the direction perpendicular to the surface. The row structure persists in these layers, and correlation of the row sequencing across steps is evident.

Some clues as to the growth mechanism of the film can be deduced from a consideration of the clean Al-Pd-Mn surface structure. The dominant motif on the clean surface is the dark pentagonal star which has been identified as a Bergman cluster (a basic structural entity of this type of quasicrystal) truncated in the surface formation process [18,25]. Grid lines superimposed on these stars have two separations of lengths $S=4.6 \pm 0.2 \AA$ and $L=$ $7.4 \pm 0.2 \AA$, i.e., identical to those found for the row structure, and are arranged in segments which form terms of the Fibonacci sequence [20,25]. The close match of the row spacings in the $\mathrm{Cu}$ structure with those of the clean surface strongly suggests that the clean surface acts as a template for growth of the row structure observed in the $\mathrm{Cu}$ film, although the detailed mechanism is unclear at this point.

For this unusual noncrystalline $\mathrm{Cu}$ structure it may be expected that there should be significant strain energy in the film and that a mechanism to reduce this strain energy 
will eventually become important. Figure 3(b) shows the structure at a coverage of $11.7 \pm 0.3 \mathrm{ML}$; in this image there is visible evidence of a high density of prominent white linear structures; some examples are indicated by arrows in Fig. 3(b). These are on average $100 \AA$ in length and $40 \AA$ in width and display a height corrugation of the order of $0.5 \AA$. Such features have been previously observed in metal on metal growth and were interpreted as partial dislocations which relieve the strain in the growing film [26]. They may also play a role in reducing the average domain size as the film thickness increases. The LEED pattern becomes streaky and diffuse and eventually degrades and disappears at a coverage equivalent to 25 layers. This is consistent with the diminishing domain size.

Upon annealing to $570 \mathrm{~K}$, desorption of the multilayer film occurs leaving an equivalent coverage of $\approx 1.5 \mathrm{ML}$ of $\mathrm{Cu}$. An irreversible structural transformation occurs to a periodic structure with five domains rotated from each other by $72^{\circ}$ (not shown). Further annealing to $660 \mathrm{~K}$ results in the desorption of the remaining $\mathrm{Cu}$ and the restoration of the LEED pattern characteristic of the clean substrate. These observations reinforce the nonequilibrium nature of the quasiperiodic film.

The discovery of this pseudomorphic $\mathrm{Cu}$ multilayer film opens up the possibility of probing the relationship between quasiperiodicity and physical properties in a single element system; for example, a depth-dependent probe such as soft $\mathrm{x}$-ray photoemission could be used to probe the electronic structure of the topmost layers close to the Fermi edge to investigate whether a pseudogap exists in such a system, as is found for conventional quasicrystals. There is also the possibility of creating further novel aperiodic nanosystems by utilizing the surface of the $\mathrm{Cu}$ film as an adsorption template. Furthermore, there is no reason to believe that the $\mathrm{Cu} / \mathrm{Al}-\mathrm{Pd}-\mathrm{Mn}$ system is unique.

The detailed mechanism for the transfer of aperiodic order from the substrate template to the adsorbate remains to be explained. The atomic structure of the $\mathrm{Cu}$ in the row system is also unknown, as is the energetic stabilization mechanism. Further studies are necessary to elucidate these and the many other intriguing questions posed by the existence of this structure.

The U.K. Engineering and Physical Sciences Research Council, the U.S. National Science Foundation, the U.S. Department of Energy, Basic Energy Sciences, and the EU Marie Curie Host Development Fund are acknowledged for funding. V. Humblot is thanked for experimental assistance, and N. M. Brabner for the construction of the $\mathrm{Cu}$ evaporator.

*Corresponding author.

Electronic address: mcgrath@liv.ac.uk
[1] L. E. Sigler, Fibonacci's Liber Abaci: A Translation into Modern English of Leonardo Pisano's Book of Calculation, Sources and Studies in the History of Mathematics and Physical Sciences (Springer, New York, 2002).

[2] The Mathematics of Long-Range Aperiodic Order, Nato Science Series C: Mathematical and Physical Sciences Vol. 489, edited by R.V. Moody (Kluwer, Dordrecht, 1995).

[3] D. Shechtman, I. Blech, D. Gratias, and J.W. Cahn, Phys. Rev. Lett. 53, 1951 (1984).

[4] The Physics of Quasicrystals, edited by P. J. Steinhardt and S. Ostlund (World Scientific, Singapore, 1987).

[5] E. Rotenberg, W. Theis, K. Horn, and P. Gille, Nature (London) 406, 602 (2000).

[6] H. R. Sharma, K. J. Franke, W. Theis, P. Gille, P. Ebert, and K. H. Rieder, Phys. Rev. B 68, 054205 (2003).

[7] E. Abe, S. J. Pennycook, and A. P. Tsai, Nature (London) 421, 347 (2003).

[8] The growth of a crystalline film which is both rotationally epitaxial and commensurate is not possible in this class of heteroepitaxial systems.

[9] A. Zangwill, Physics at Surfaces (Cambridge University Press, Cambridge, 1988).

[10] V. Fournée, T. C. Cai, A. R. Ross, T. A. Lograsso, J.W. Evans, and P. A. Thiel, Phys. Rev. B 67, 033406 (2003).

[11] B. Bolliger, V. E. Dmitrienko, M. Erbudak, R. Luscher, H. U. Nissen, and A. R. Kortan, Phys. Rev. B 63, 052203 (2001).

[12] T. Cai, J. Ledieu, V. Fournée, T. Lograsso, A. Ross, R. McGrath, and P. A. Thiel, Surf. Sci. 526, 115 (2003).

[13] K. J. Franke, H. R. Sharma, W. Theis, P. Gille, P. Ebert, and K. H. Rieder, Phys. Rev. Lett. 89, 156104 (2002).

[14] M. Shimoda, J. Q. Guo, T. J. Sato, and A. P. Tsai, Jpn. J. Appl. Phys. 40, 6073 (2001).

[15] A. R. Smith, K-J. Chao, Q. Niu, and C K. Shih, Science 273, 226 (1996).

[16] P. Ebert, K.-J. Chao, Q. Niu, and C. K. Shih, Phys. Rev. Lett. 83, 3222 (1999).

[17] J. Ledieu, R. McGrath, R. D. Diehl, T. A. Lograsso, D.W. Delaney, Z. Papadopolos, and G. Kasner, Surf. Sci. 492, L729 (2001).

[18] Z. Papadopolos, G. Kasner, R. D. Diehl, J. Ledieu, E. J. Cox, N.V. Richardson, Q. Chen, T. A. Lograsso, A. R. Ross, and R. McGrath, Phys. Rev. B 66, 184207 (2002).

[19] L. Barbier, D. Le Floc'h, Y. Calvayrac, and D. Gratias, Phys. Rev. Lett. 88, 085506 (2002).

[20] T. M. Schaub, D. E. Bürgler, H. J. Güntherodt, and J. B. Sück, Phys. Rev. Lett. 73, 1255 (1994).

[21] H. Asonen, C. J. Barnes, A. Salokatve, and M. Pessa, Surf. Sci. 152/153, 262 (1985).

[22] C. J. Barnes, H. Asonen, A. Salokatve, and M. Pessa, Surf. Sci. 184, 163 (1987).

[23] P. Ebert, F. Yue, and K. Urban, Phys. Rev. B 57, 2821 (1998).

[24] R. D. Diehl, J. Ledieu, N. Ferralis, A.W. Szmodis, and R. McGrath, J. Phys. Condens. Matter 15, R63 (2003).

[25] J. Ledieu and R. McGrath, J. Phys. Condens. Matter 15, S3113 (2003).

[26] V. Fournée, J. Ledieu, T. Cai, and P. A. Thiel, Phys. Rev. B 67, 155401 (2003). 